\title{
miR-28-5p promotes the development and progression of ovarian cancer through inhibition of N4BP1
}

\author{
JUAN XU* ${ }^{*}$ NAN JIANG ${ }^{*}$, HAIJUAN SHI, SHANSHAN ZHAO, SHUZHONG YAO and HUIMIN SHEN \\ The First Affiliated Hospital, Sun Yat-sen University, Guangzhou, Guangdong 510080, P.R. China
}

Received October 10, 2016; Accepted February 21, 2017

DOI: 10.3892/ijo.2017.3915

\begin{abstract}
MicroRNAs (miRNAs) play important roles in transcriptional regulation by targeting the 3'-UTR of target genes which participate in various biological processes. We aimed to investigate the potential role of miR-28-5p in the process of ovarian cancer development through regulating N4BP1. We found that the mRNA expression level of miR-28-5p was significantly increased in ovarian cancer tissues in comparison with adjacent ovarian tissues by qRT-PCR $(\mathrm{P}<0.0001)$. We established that miR-28-5p promoted the progression of ovarian cancer cell proliferation using colony forming assay and MTT assay. Wound healing assay and the migration and invasion assay showed that miR-28-5p accelerated the migration and invasion abilities of ovarian cancer cells. Simultaneously, we showed that miR-28-5p promoted ovarian cancer cell cycle, and inhibited apoptosis by flow cytometry in vitro. Furthermore, the results showed that miR-28-5p promoted the growth of ovarian tumor by tumor formation assay in vivo. The results of western blot analysis indicated that miR-28-5p promoted the protein expression level of F-actin. Western blot analysis also demonstrated that miR-28-5p promoted the progress of epithelial-mesenchymal transition (EMT) in ovarian carcinoma cells. In addition, we found that miR-28-5p downregulated N4BP1 mRNA and protein expression by qRT-PCR and western blot analysis in human ovarian cancer. Therefore, our study indicated that miR-28-5p promoted the progression of ovarian cancer cell cycle, proliferation, migration and invasion, inhibited apoptosis, and induced the process of EMT through inhibition of N4BP1 in vitro. Moreover, miR-28-5p promoted the growth of ovarian tumor in vivo.
\end{abstract}

Correspondence to: Dr Huimin Shen or Dr Shuzhong Yao, The First Affiliated Hospital, Sun Yat-sen University, No. 58 Zhongshan II Road, Guangzhou, Guangdong 510080, P.R. China

E-mail: shenhuimin0505@163.com

E-mail: yaoshuzhong1062@163.com

${ }^{*}$ Contributed equally

Key words: miR-28-5p, N4BP1, ovarian cancer, epithelial-mesenchymal transition

\section{Introduction}

Ovarian cancer, one of the most common malignant tumors, takes the top spot among all gynecologic cancers with the high mortality, and is still a serious threat to women's life in China in the next few decades $(1,2)$. At present, the combination therapy combining surgery, radiotherapy, and chemotherapy were mostly adopted for the treatment of ovarian cancer. Unfortunately, as a result of no early symptoms and effective biomarker screening, the limitation of diagnosis technology, $>70 \%$ of the patients with ovarian cancer have late-stage, accompanied by distant metastases when they are diagnosed (3-5). Therefore, the molecular mechanism research on the development mechanisms of ovarian cancer are extremely urgent.

MicroRNAs (miRNAs), are a major kind of endogenous non-coding small RNAs with $\sim 20$ nucleotides, participating in post-transcriptional regulation to affect the biological processes by targeting the 3'-UTR of target genes $(3,6-10)$. Many studies have shown that miRNAs, serving as a kind of regulatory genes, have played important roles in the occurrence and development of various diseases including the progression and tumorigenesis of human cancers $(4,8,11,12)$. Numerous studies have indicated that various miRNAs were relative to the disease progression of ovarian cancer $(3,13-16)$. The mechanism and function of miR-28-5p have been studied in VHL-associated cancer (17), colorectal cancer (18), and breast cancer (19). However, the functional relevance of miR-28-5p in ovarian cancer is unclear.

Epithelial-mesenchymal transition (EMT) is the biological processe where epithelial cells are transformed into mesenchymal cells (20-22). Numerous studies have indicated that the adhesion ability of tumor cells will decrease the migration capacity of tumor cells. Thus, EMT was relative to metastasis of tumors. In the process of EMT, the expression level of cell adhesion molecules (E-cadherin) was decreased; the mesenchymal markers (vimentin) was increased (23). As is known, F-actin (filamentous) is essential for important cellular functions, such as the mobility and contraction of cells and participating in the process of cell migration (24).

N4BP1 is involved in the normal development by interacting with the WW and HECT domains of the E3 ubiquitin ligase Nedd4 and participating in the process to find targets of ubiquitin-mediated protein degradation (25). It has been indicated that N4BP1 was related to the related E3 ligase 
ITCH (26) and was combined with ITCH to negatively regulate ITCH E3 activity by binding to its substrates such as p73 and p63, and c-Jun (27). At present, the function of N4BP1 is unclear, but it has been reported that N4BP1 may have ribonuclease activity accoording to YacP-like nuclease (NYN) domain, as characterized nucleases (28). Research has shown that miR-28-5p negatively regulated the expression level of N4BP1 dual luciferase reporter gene assay. Our present study demonstrated the effects of miR-28-5p in ovarian cancer, the functional mechanism between miR-28-5p and N4BP1, and the potential downstream genes potentially regulating these processes, also demonstrating that miR-28-5p could be a potential therapeutic target for treatment of ovarian cancer.

\section{Materials and methods}

Cell lines and transfection. Human epithelial ovarian cancer cell lines ES2 were obtained from American Type Culture Collection (ATCC, Manassas, VA, USA) and SKOV3 cells were purchased from College of Life Science, Hunan Normal University, China. ES2 and SKOV3 cells were cultured in Dulbecco's modified Eagle's medium (DMEM) (Invitrogen, Carlsbad, CA, USA) including 10\% fetal bovine serum (FBS; Invitrogen), penicillin (100 U/ml), and streptomycin $(100 \mu \mathrm{g} / \mathrm{ml})$ at $37^{\circ} \mathrm{C}$ and $5 \% \mathrm{CO}_{2}$. For the treatment, $2 \times 10^{5} \mathrm{ES} 2$ or SKOV3 cells were cultured in 6-well plates and then transfected with $200 \mu 1$ mature miR-28-5p mock, mimic, or inhibitor (GenePharma Co., Ltd., Shanghai, China) for $72 \mathrm{~h}$. All transfections were completed using Lipofectamine ${ }^{\text {TM }} 3000$ (Invitrogen) according to the manufacturer's protocols.

Clinical specimens. In this study, the ovarian cancer tissues and adjacent non-cancerous tissues samples $(5 \mathrm{~cm}$ from the edge of the cancer) were collected from the First Affiliated Hospital of Sun Yat-sen University between 2015 and 2016, and this study obtained Ethics committee approval of the First Affiliated Hospital of Sun Yat-sen University. Informed consent was also obtained from each patient. The ovarian cancer histological diagnosis was assessed on the basis of the World Health Organization (WHO). All tissue samples were stored at $-80^{\circ} \mathrm{C}$.

RNA reverse transcription. Total RNA was extracted from ovarian cancer tissues, matched adjacent non-cancerous tissues and treated ES2 and SKOV3 cells using the TRIzol reagent (Invitrogen). The RevertAid First Strand cDNA Synthesis kit (Thermo Fisher Scientific) was used to synthesize cDNAs with random primers according to the manufacturer's protocols.

Quantitative real-time reverse transcription PCR ( $q R T-P C R)$. As described previously (29), the mRNA expression levels were detected using the SYBR-Green PCR Master Mix kit (Takara) in PCR reaction. The primer sequences for GAPDH are: 5'-TGTTCGTCATGGGTGTGAAC-3' (forward) and 5'-ATG GCATGGACTGTGGTCAT-3' (reverse) (internal control). The primer sequences for N4BP1 are: 5'-TATGCAGCCCCTA CTCAGTG-3' (forward) and 5'-GCTCGTTGGTTTCTGCA GAA-3' (reverse). The primer sequences for hsa-miR-28-5p are: 5'-AAGGAGCUCACAGUCUAUUGAG-3'. The primer sequences for U6 are: 5'-CTCGCTTCGGCAGCACA-3' (forward) and 5'-AACGCTTCACGAATTTGCGT-3' (reverse). All data are reported as the mean $\pm \mathrm{SD}$ of three independent experiments.

Western blot analysis. Seventy-two hours after mature miR-28-5p mock, mimic, or inhibitor transfection, the ES2 and SKOV3 cells were lysed using lysis buffer containing a protease inhibitor cocktail (P8340; Sigma-Aldrich, St. Louis, MO, USA). The concentrations of total proteins were analyzed using BCA Protein Assay kit (Thermo Fisher Scientific, Rockford, IL, USA). Proteins ( $30 \mu \mathrm{g})$ were added into each lane on the $8 \%$ SDS/PAGE gels based on the molecular weight of the objective proteins. The $5 \%$ skim milk (BD Biosciences) was used to block the PVDF membranes, and then they were (Millipore, Billerica, MA, USA) used to incubate the optimal concentration of primary antibody at $4^{\circ} \mathrm{C}$ overnight. The next day, the horseradish peroxidaseconjugated secondary antibodies with a proper dilution were incubated for $1 \mathrm{~h}$ at room temperature. The experimental results were visualized using the enhanced chemiluminescence (ECL) substrate kit (Amersham Biosciences, Inc., Piscataway, NJ, USA) and the enhanced chemiluminescence detection system (Amersham Biosciences). The primary antibodies used were the anti-N4BP1 antibody (rabbit, 1:100, Abcam, Cambridge, MA, USA); the anti-F-actin (1:200, Cell Signaling Technology, Boston, MA, USA); the antiE-cadherin (1:5,000, BD Biosciences, San Jose, CA, USA); the anti-vimentin $(1: 1,000$, V6630, Sigma-Aldrich); the anti-GAPDH antibody (1:4,000, Cell Signaling Technology, Beverly, MA, USA) was used as internal control.

Immunohistochemistry (IHC) assay. According to the manufacturer's instructions, immunohistochemistry of N4BP1 was completed on 5-mm formalin fixed; paraffin-embedded tissue sections which were cut from the FFPE blocks. The sections were incubated with rabbit anti-N4BP1 antibody (Abcam) at $4^{\circ} \mathrm{C}$ overnight. The automated immunostainer (Ventana Medical Systems, Tucson, AZ, USA) was used to complete the immunohistochemical stains. The N4BP1 staining results were classified as 0 , negative staining; 1 , weak staining; 2 , moderate staining; and 3 , intense staining. The negative staining and weak staining cells were considered as low expressors, and the moderate staining and intense staining cells were considered to be high expressors.

Colony forming unit (CFU) assay. The ES2 or SKOV3 cells which were transfected with miR-28-5p mock, mimics, or inhibitors were seeded into the DMEM complete medium for 7 days. Then the colons were fixed using methanol for $15 \mathrm{~min}$, and dyed using Giemsa dye solution for $10 \mathrm{~min}$. Then the colony forming units were recorded and counted.

MTT assay. The treated ES2 or SKOV3 cells (2,000 cells/well) were seeded in 96-well plates with complete medium for 24 , 48 and $72 \mathrm{~h}$, respectively. The 3-(4,5-dimethylthiazol-2-yl)2,5-diphenyltetrazolium bromide (MTT, $20 \mu \mathrm{l} /$ well) solution $(5 \mathrm{mg} / \mathrm{ml})$ was added into each well at the particular point in time. After $4 \mathrm{~h}, 100 \mu 1$ dimethyl sulfoxide solutions were added to dissolve the crystal. The absorbance was detected using a 


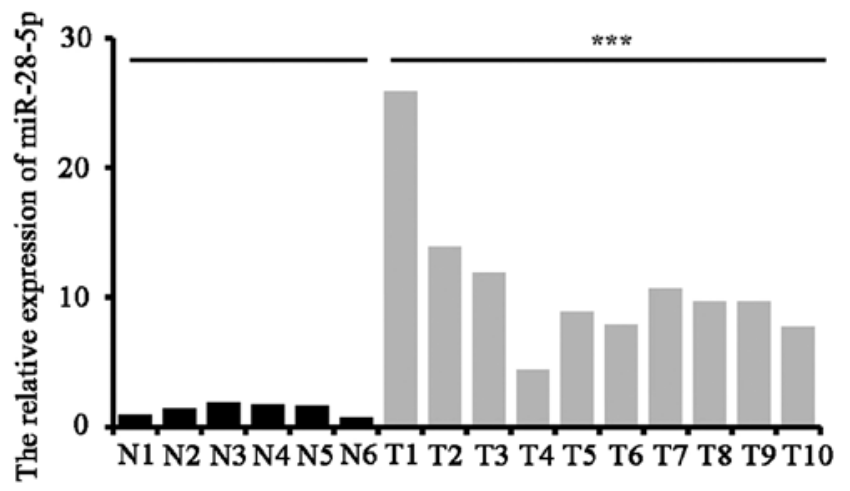

Figure 1. miR-28-5p overexpression in ovarian cancer tissues. qRT-PCR was used to detect the mRNA expression level of miR-28-5p in ovarian cancer tissues $(n=10)$ and adjacent ovarian tissues $(n=6) .{ }^{* * *} P<0.001$.

micro-plate reader (Bio Tek Instruments, Inc., Winooski, VT, USA) at $490 \mathrm{~nm}$.

Flow cytometric analysis of the cell cycle. The treated ES2 or SKOV3 cells were resuspended in PBS containing $70 \%$ ethanol, $0.5 \mathrm{mg} / \mathrm{ml} \mathrm{RNase} \mathrm{A}$ and $0.1 \mathrm{mg} / \mathrm{ml}$ propidium iodide. The images of cell cycle were obtained using the FACSCalibur (BD Biosciences). The results were analyzed using FlowJo software (Tree Star Corp., Ashland, OR, USA).

Flow cytometric analysis of the cell apoptosis. Likewise, the treated ES2 or SKOV3 cell suspension was stained with FITC-Annexin V and propidium iodide (PI). The flow cytometry results were analyzed using FlowJo software. Cells were segmented into four types, such as the viable cells, dead cells, the early stage apoptotic cells, the late stage apoptotic cells, and the dead cells.

Wound healing assay. As described previously (30), the treated ES2 or SKOV3 cells were incubated in $60-\mathrm{mm}$ culture plates, and the small linear wounds were created. The cell debris was washed off gently. After $24 \mathrm{~h}$, the results were analyzed by image analysis software (National Institute of Health, Bethesda, MD, USA). The degree of wound healing depended on the distance of migration of cells from the edge of the scratch.

Migration and invasion assays. According to the manufacturer's instructions, the migration capacity of the treated ES2 or SKOV3 cells were evaluated in 24-well Transwell cell culture chamber (costar). Treated cells $(200 \mu \mathrm{l})\left(2.5 \times 10^{5} / 100 \mu \mathrm{l}\right)$ were incubated in serum-free media and added into the upper chamber of the $8-\mu \mathrm{m}$ pore cell culture inserts, and complete medium with $10 \%$ FBS was added to the lower chambers. After $24 \mathrm{~h}$, the migratory cells were fixed by $4 \%$ paraformaldehyde, and stained by $0.1 \%$ crystal violet solution. The number of migratory cells was counted using a 20X objective microscope. For the invasion assay, $10 \mu 1$ 1:8 diluted Matrigel (BD Biosciences, San Diego, CA, USA) was pre-paved into the polycarbonate membrane of Transwell inserts before the experiment at $37^{\circ} \mathrm{C}$ for $2 \mathrm{~h}$.

Tumor formation in nude mice. The animal experiments were approved by the Institutional Committee for Animal
Research and performed according to the Institutional Animal Care and Use Committee. The flanks of 5-week-old BALB/c athymic nude mice were injected subcutaneously with the ES2 cells treated with negative control or miR-28-5p agomir $\left(1 \times 10^{7}\right.$ cells in $\left.100 \mu \mathrm{l}\right) 5$ times for 4 weeks. At the planned time every week, the mice were sacrificed, the bodies were dissected, the tumor weight was measured and the tumor volume was calculated based on the formula: $\mathrm{V}=\pi \mathrm{AB}^{2} / 6$, where $\mathrm{A}$ means the largest diameter and $\mathrm{B}$ means the perpendicular diameter.

Statistical analysis. The data were analyzed by the Student's t-test and variance (ANOVA) using SPSS 15.0 software (SPSS, Chicago, IL, USA). All results are expressed as means $\pm \mathrm{SE}$. The statistical significance was set at $\mathrm{P}<0.05$.

\section{Results}

miR-28-5p overexpression in ovarian cancer tissues. We used the ovarian cancer tissues from 10 patients and adjacent noncancerous tissues from 6 patients randomly. The expression level of miR-28-5p was measured by qRT-PCR. The results indicated that the expression level of miR-28-5p was increased in ovarian cancer tissues $(n=10)$ compared with adjacent noncancerous tissues $(\mathrm{n}=6)(\mathrm{P}<0.001)$ (Fig. 1).

miR-28-5p promotes the progression of cell cycle and proliferation and inhibits apoptosis in ovarian cancer cells. The impacts of miR-28-5p expression levels on the ability of ovarian cancer cell proliferation, cycle, and apoptosis was detected in ES2 or SKOV3 cells which were transfected with mature miR-28-5p mock, mimic, or inhibitor, respectively. The colony forming unit assay was used to measure the ovarian cell proliferation ability. Our results found that the proliferation ability was significantly increased in ES2 or SKOV3 cells transfected with mature miR-28-5p mimic compared with the control group (mock) $(\mathrm{P}<0.001)$. The proliferation ability was significantly decreased in ES2 or SKOV3 cells transfected with mature miR-28-5p inhibitor compared with the control group (mock) $(\mathrm{P}<0.001)$ (Fig. 2A). Similarly, the ovarian cell proliferation ability also was detected by MTT assay, and the results showed that miR-28-5p promoted the proliferation capacity of ES2 or SKOV3 cells (Fig. 2B). We found that the cell cycle significantly arrested in G1/G0 in ES2 cells transfected with mature miR-28-5p inhibitor (Diploid, 100.00\%; Dip G1/ G0, 82.45\%; Dip S, 8.90\%; Dip G2/M, 8.65\%) compared with the control group (Diploid, 100.00\%; Dip G1/G0, 69.55\%; Dip S, 26.08\%; Dip G2/M, 4.37\%) by flow cytometry; and the cell cycle significantly arrested in G1/G0 in SKOV3 cells transfected with mature miR-28-5p inhibitor (Diploid, 100.00\%; Dip G1/G0, 84.44\%; Dip S, 7.98\%; Dip G2/M, 7.58\%) compared with the control group (Diploid, 100.00\%; Dip G1/ G0, 70.81\%; Dip S, 22.75\%; Dip G2/M, 6.44\%) (Fig. 2C). The cell apoptosis capacity of ES2 and SKOV3 cells transfected with mature miR-28-5p inhibitor was significantly increased compared with the control group by Annexin V-FITC/PI staining $(\mathrm{P}<0.001)$. The cell apoptosis capacity of ES2 and SKOV3 cells transfected with mature miR-28-5p mimic was significantly decreased compared with the control group $(\mathrm{P}<0.001)$ (Fig. 2D). 
A
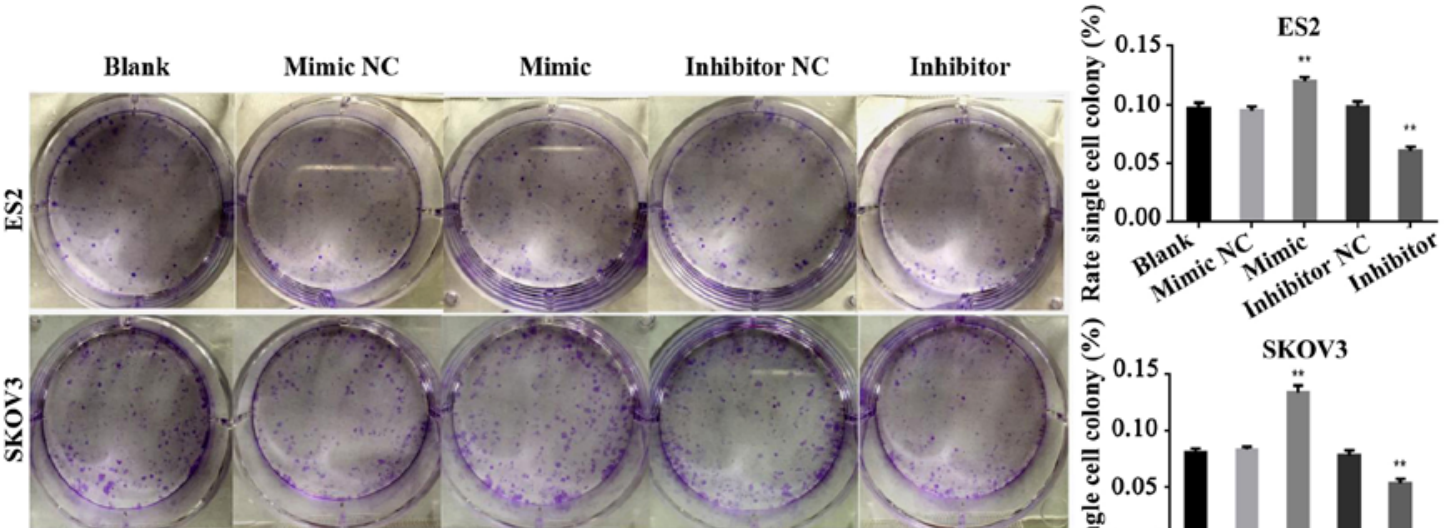

B

ES2
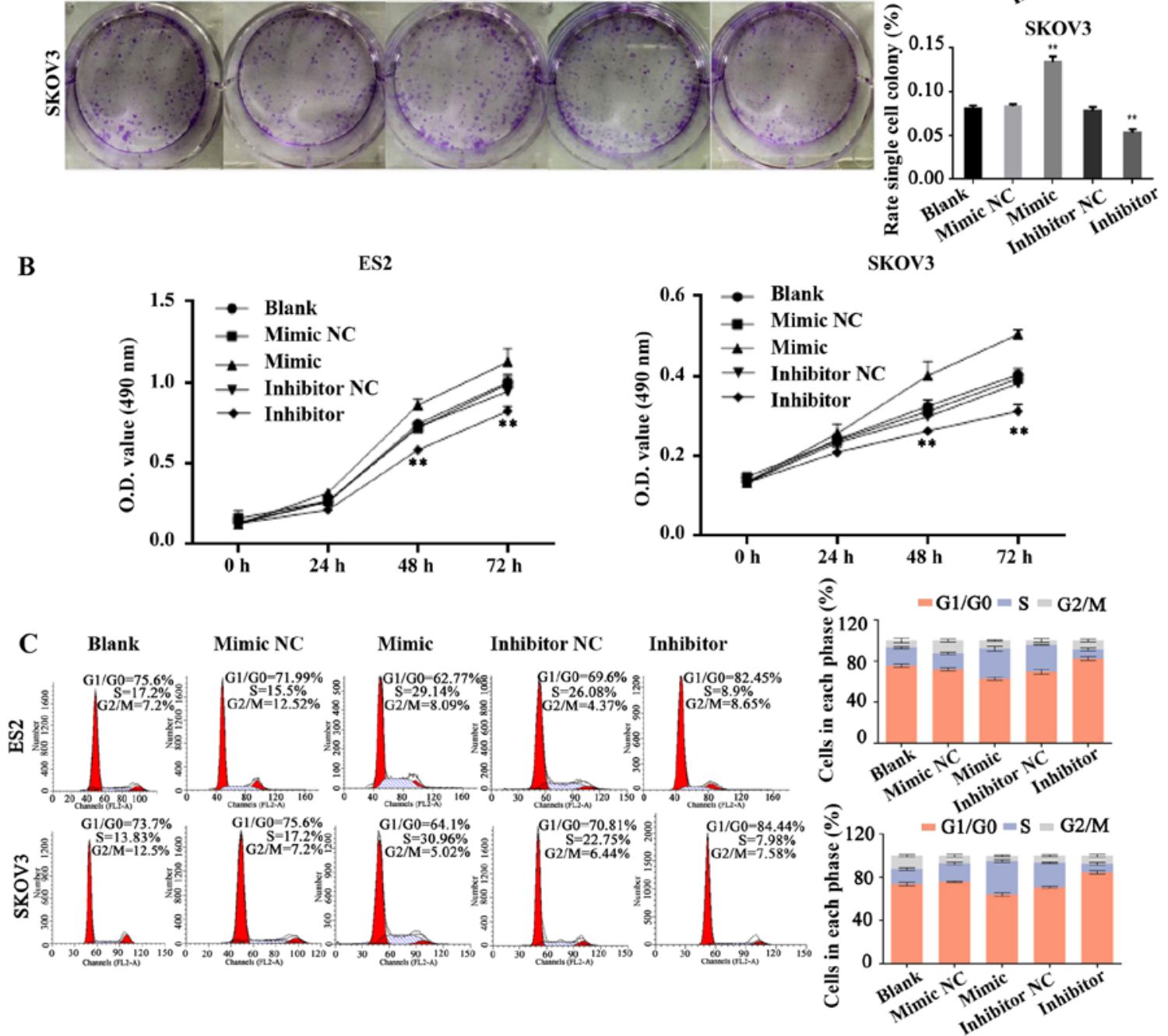

D
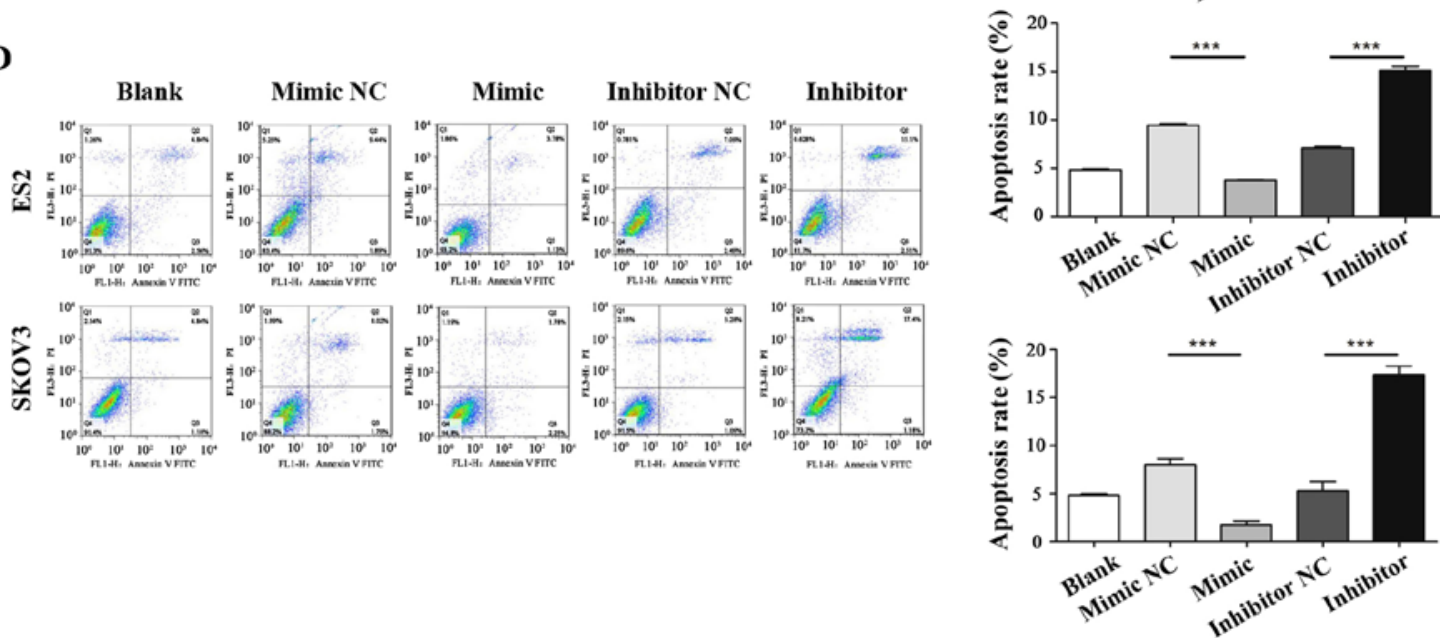

Figure 2. miR-28-5p promotes the progression of cell cycle and proliferation and inhibits apoptosis in ovarian cancer cells. (A) The ability of cell proliferation was detected by clonal colony forming experiment in ES2 and SKOV3 transfected with miR-28-5p mimic, inhibitor or negative control (NC) respectively $\left({ }^{* *} \mathrm{P}<0.01\right)$. (B) MTT assay was performed to determine cell proliferation ability in ES2 and SKOV3 transfected with miR-28-5p mimic, inhibitor or NC respectively. The values of expression level are represented by the mean percent of means $\pm \mathrm{SD}\left(\mathrm{n}=3,{ }^{* *} \mathrm{P}<0.01\right)$. (C) The cell cycle distribution was detected by flow cytometry. (D) Ovarian cancer cells were transfected with miR-28-5p mimic, inhibitor or NC and cell apoptosis was detected by Annexin V-FITC/PI staining $\left({ }^{* * *} \mathrm{P}<0.001\right)$ 
A
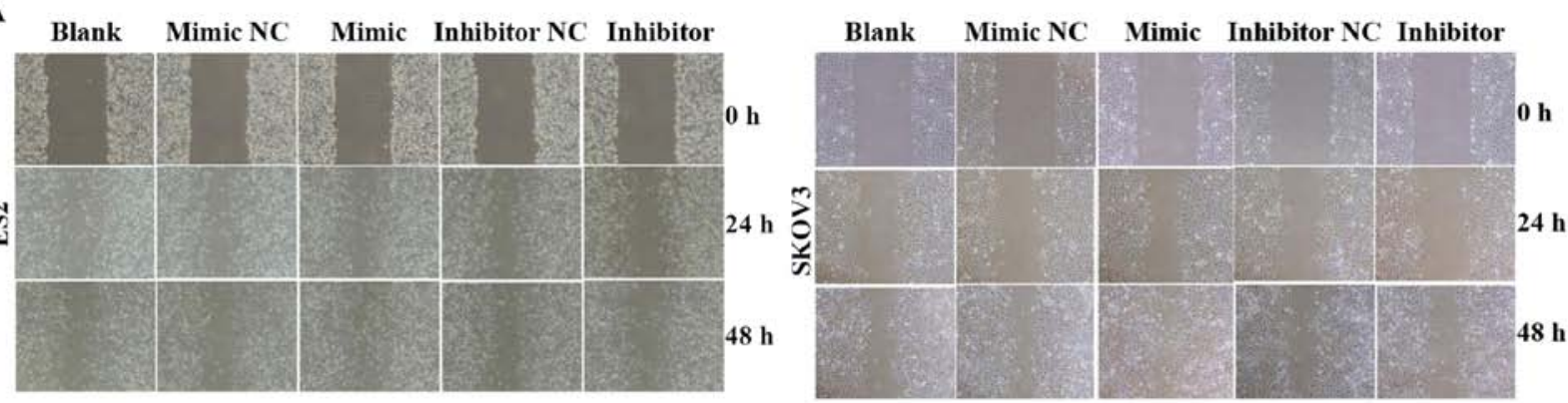

B

Migration assay
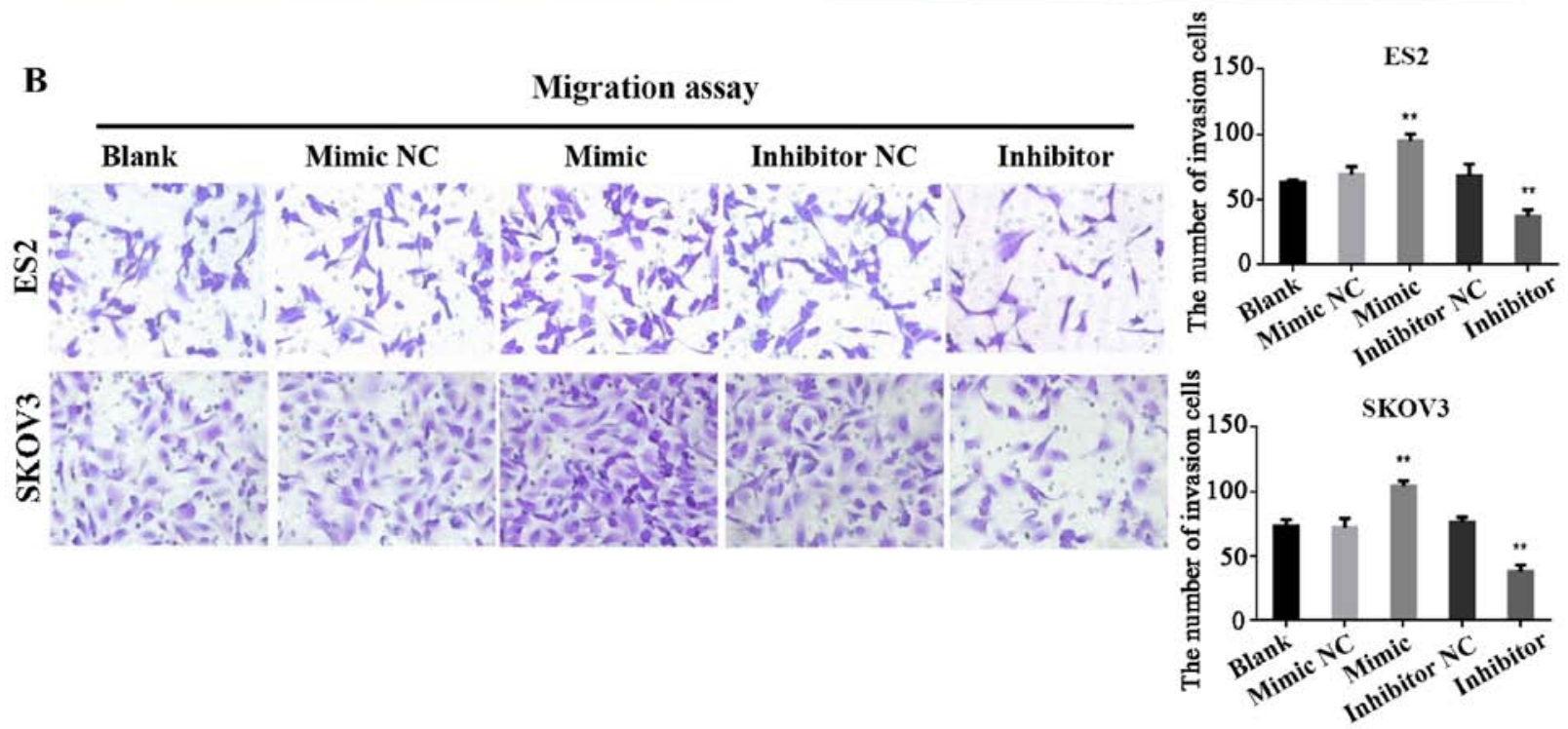

C

Invasion assay
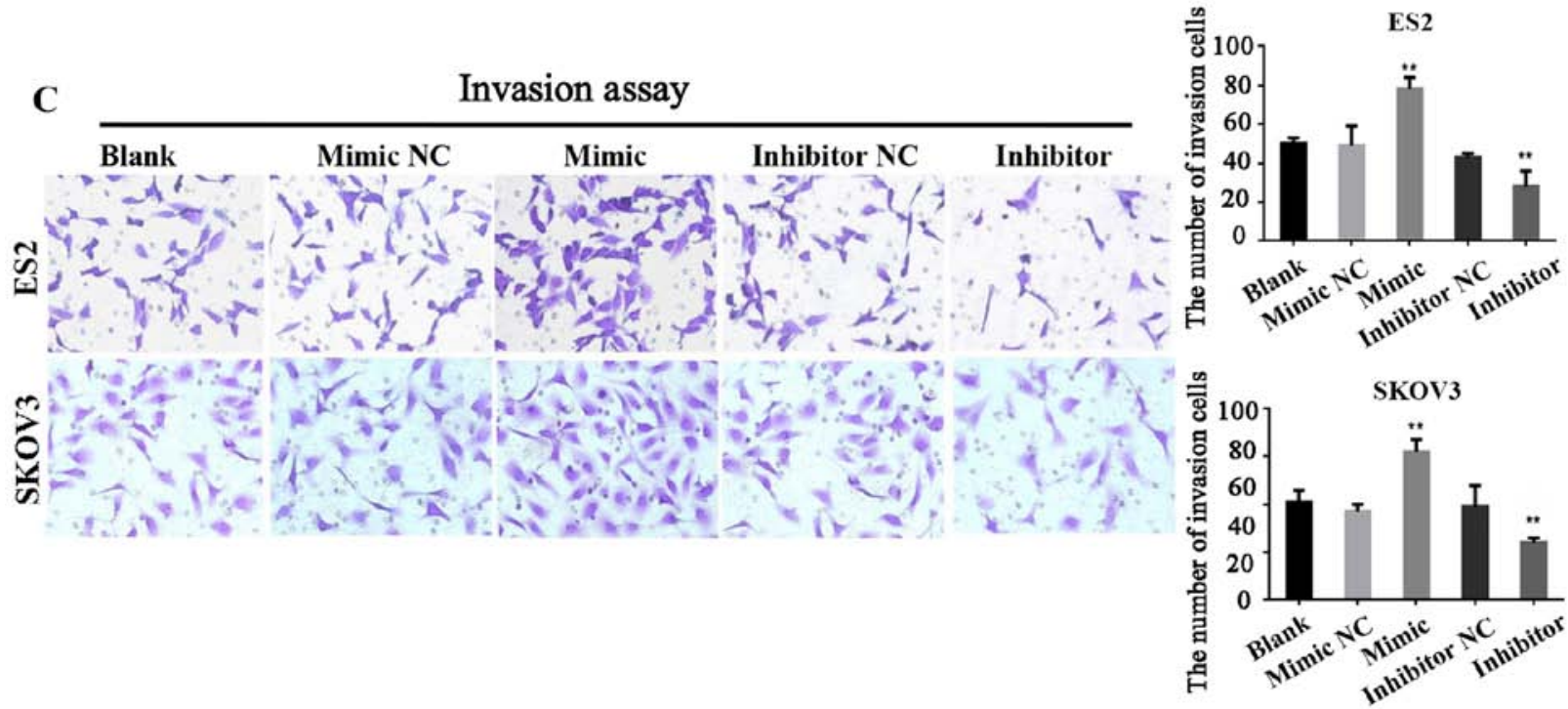

Figure 3. miR-28-5p expression accelerates migration and invasion ability of ovarian cancer cells. (A) The migration ability of ES2 and SKOV3 cells transfected with miR-28-5p mimic, inhibitor or NC were measured by wound healing assay at 0, 24 and $48 \mathrm{~h}$ respectively. (B) The migration assays of ES2 and SKOV3 cells transfected with miR-28-5p mimic, inhibitor or NC respectively were performed. Magnification, $\mathrm{x} 200$; scale bars, $10 \mu \mathrm{m}\left({ }^{* *} \mathrm{P}<0.01\right)$. $(\mathrm{C}) \mathrm{The}$ invasion assays of ES2 and SKOV3 cells transfected with miR-28-5p mimic, inhibitor or NC respectively were performed. Magnification, x200; scale bars, $10 \mu \mathrm{m}\left({ }^{* *} \mathrm{P}<0.01\right)$.

miR-28-5p expressions accelerate migration and invasion ability of ovarian cancer cells. We further studied the cell migration and invasion ability which was affected by miR-28-5p in ovarian cancer cells. Firstly, the migration ability of ES2 and SKOV3 cells transfected with miR-28-5p mimic, inhibitor or NC were measured by wound healing assay at 0 , 24 and $48 \mathrm{~h}$, respectively. The results indicated that the cell migration capacity of ES2 and SKOV3 cells transfected with mature miR-28-5p mimic was significantly increased compared with the control group $(\mathrm{P}<0.01)$; the cell migration capacity of ES2 and SKOV3 cells transfected with mature miR-28-5p inhibitor was significantly decreased compared with the control group $(\mathrm{P}<0.01)$ (Fig. 3A). Secondly, the migration ability was detected using the migration assay. The results also showed 


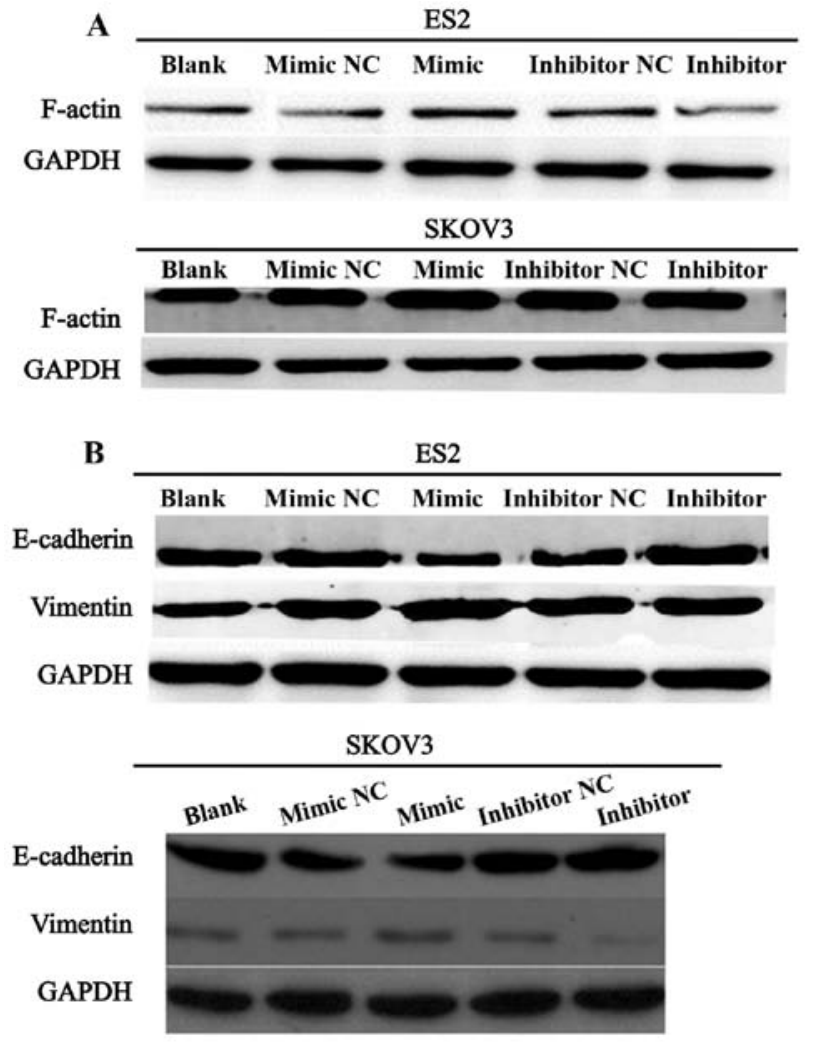

Figure 4. miR-28-5p regulates F-actin, E-cadherin, and vimentin expression. (A) Western blot analysis detected the expression level of F-actin in ES2 and SKOV3 cells transfected with miR-28-5p mimic, inhibitor or NC. GAPDH was used as a protein-loading control. (B) The expression levels of E-cadherin and vimentin were measured by western blot analysis in ES2 and SKOV3 cells transfected with miR-28-5p mimic, inhibitor or NC.

that miR-28-5p expression accelerated the migration ability of ovarian cancer cells (Fig. 3B). Finally, the results of the invasion assay also indicated that the cell invasion ability of ES2 and SKOV3 cells transfected with mature miR-28-5p mimic was significantly increased compared with the control group $(\mathrm{P}<0.01)$; the cell invasion capacity of ES2 cells and SKOV3 transfected with mature miR-28-5p inhibitor was significantly decreased compared with the control group $(\mathrm{P}<0.01)$ (Fig. 3C).

miR-28-5p regulates $F$-actin, E-cadherin, and vimentin expression. We further investigated whether miR-28-5p expression influenced the protein expression level of F-actin. Western blot assay was performed to detect the protein expression level of F-actin in ES2 and SKOV3 cells transfected with miR-28-5p mimic, inhibitor or NC. As showed in Fig. 4A, the expression level of F-actin was significantly upregulated in ES2 and SKOV3 cells transfected with mature miR-28-5p mimic compared with the control group. Conversely, F-actin expression was significantly downregulated in ES2 and SKOV3 cells transfected with mature miR-28-5p inhibitor in comparison with the control group. Furthermore, we measured the expression levels of proteins associated with epithelial-mesenchymal transition (EMT) in ES2 and SKOV3 cells transfected with miR-28-5p mimic, inhibitor or NC. The results indicated that miR-28-5p downregulated the protein expression level of E-cadhetin and upregulated the protein expression level of vimentin in ES2 and SKOV3 cells (Fig. 4B). Therefore, our investigation demonstrated that miR-28-5p promotes the progress of EMT in ovarian carcinoma cells.

miR-28-5p downregulates N4BPI expression in human ovarian cancer. To explore the protein expression level of N4BP1 in human ovarian cancer tissues, we performed IHC analysis of the human ovarian cancer tissues. The results indicated that the expression of N4BP1 was lower in ovarian cancer tissues compared with normal ovarian tissues. Therefore, we concluded that N4BP1 has low expression in human ovarian cancer (Fig. 5A). To further obtain the mechanism of miR-28-5p in ovarian cancer, we studied the relationship between miR-28-5p and N4BP1. The qRT-PCR results revealed that miR-28-5p inhibited the mRNA expression level of N4BP1 in ES2 and SKOV3 cells (Fig. 5B). We also detected the protein expression level of N4BP1 using western blot analysis. We found that miR-28-5p inhibited the protein expression level of N4BP1 in ES2 and SKOV3 cells (Fig. 5C). Therefore, we proved that miR-28-5p downregulated N4BP1 expression in human ovarian cancer.

miR-28-5p promotes the growth of ovarian tumor in vivo. To assess the effect of miR-28-5p on tumorigenesis in vivo, ES2-blank, ES2-NC, and ES2-agomir cells were implanted subcutaneously into nude mice. The mice were sacrificed at 4, 9, 15, 21 and 28 days respectively (Fig. 6A), the bodies were dissected, and the tumor removed (Fig. 6B). Tumor weight was measured and tumor volume was calculated. The results showed that mice injected with ES2-agomir cells developed larger tumors than those injected with ES2-blank, or ES2-NC cells $(\mathrm{P}<0.01)$ (Fig. 6C and D).

\section{Discussion}

Ovarian cancer is one of the most universal cancers in women and ranks the sixth in female cancers in the world resulting in $\sim 125,000$ deaths each year (31). Due to technical limitations, the patients have advanced ovarian carcinoma when they are diagnosed. There are only $30 \%$ of advanced-stage ovarian cancer patients who can survive $>5$ years after diagnosis (32). Therefore, it is urgent to study the pathological mechanism in ovarian cancer for exploiting new diagnosis and new treating method against this malignancy.

MicroRNAs (miRNAs), a class of small non-coding RNAs, regulate mRNAs. Many studies showed that miRNAs have important functions, such as cell proliferation, metastasis, inflammation, and angiogenesis of tumor by targeting mRNAs in various cancers $(33,34)$. Many studies have indicated that miRNAs are closely related to the development and progression of diverse diseases $(8,11,12)$. There are reports that various miRNAs such as miR-141, miR-200a, miR-199a, miR-223, miR-100, and miR-9 are associated with the development process of ovarian cancer (15,35-37). The mechanism and function of miR-28-5p have been proven in colorectal cancer (18) and hepatocellular carcinoma $(38,39)$. However, the functional relevance of miR-28-5p in ovarian cancer is still not known. In our study, we found that miR-28-5p had higher expression in ovarian cancer tissues in comparison with adjacent ovarian tissues. miR-28-5p promoted the progression of ovarian cancer cell cycle, proliferation, migration and invasion, and inhibited 
A

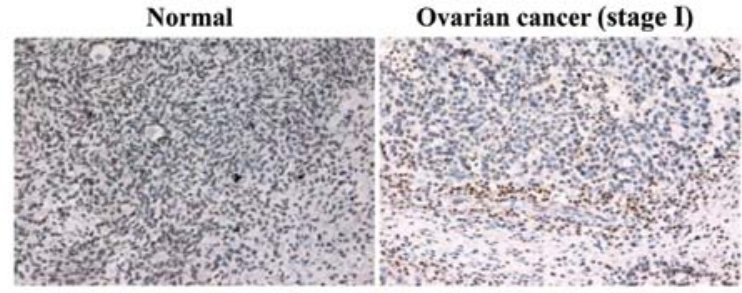

Ovarian cancer (stage II) Ovarian cancer (stage III-IV)

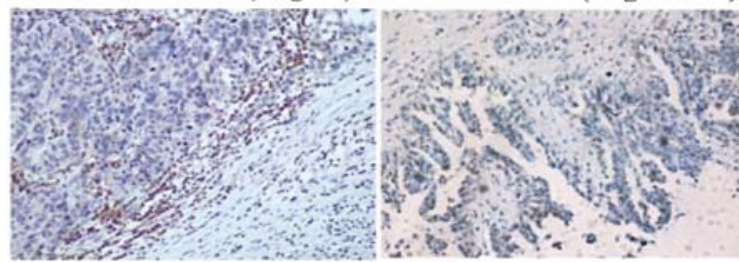

B
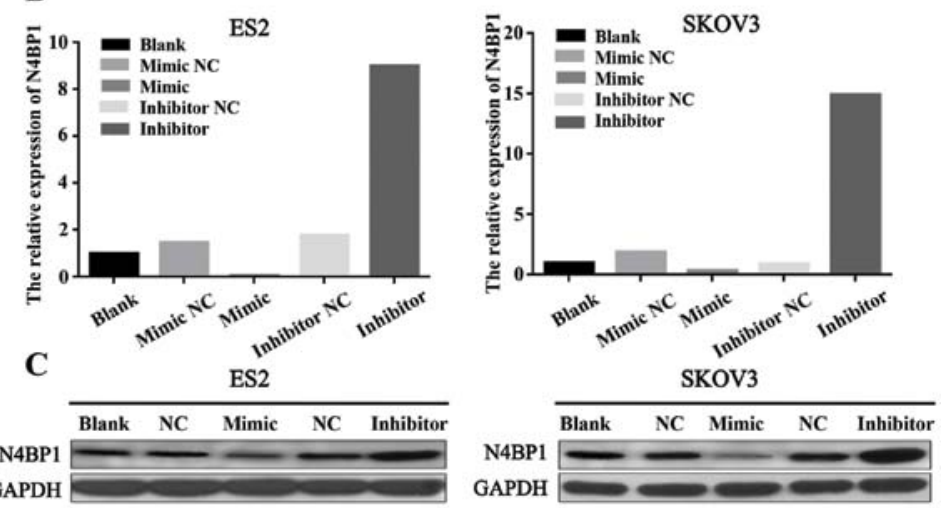

Figure 5. miR-28-5p downregulates N4BP1 expression in human ovarian cancer. (A) Representative images of N4BP1 immunohistochemical staining in ovarian cancer tissues and adjacent ovarian tissues, original magnification, $x 400$. (B) miR-28-5p inhibited the mRNA expression level of N4BP1 in ES2 cells. The mRNA expression level of N4BP1 was detected by qRT-PCR in ES2 and SKOV3 cells transfected with miR-28-5p mimic, inhibitor or NC. (C) The expression level of N4BP1 was detected by western blot analysis in ES2 and SKOV3 cells transfected with miR-28-5p mimic, inhibitor or NC.

$\mathbf{A}$

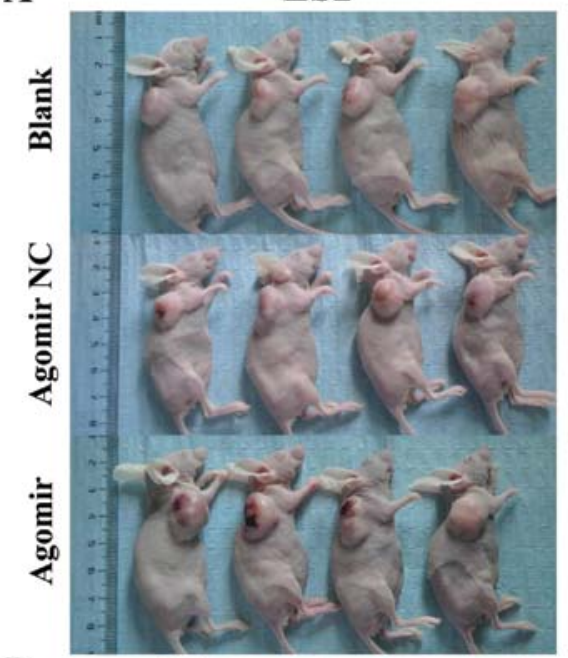

B

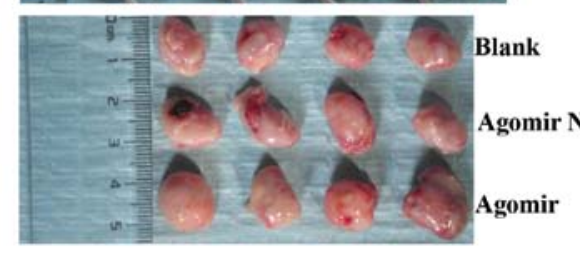

C

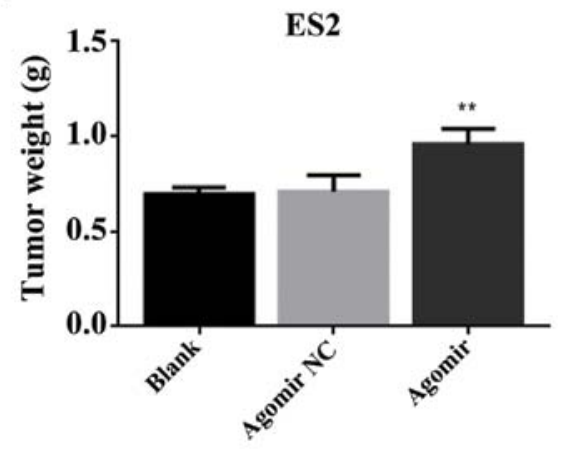

D

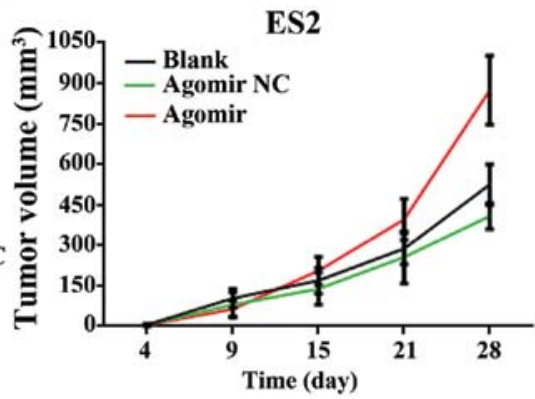

Figure 6. miR-28-5p promotes the growth of ovarian tumor in vivo. (A) miR-28-5p agomir treatment decreased the size of tumor induced in nude mice. Athymic mice were treated 5 times for 4 weeks with $1 \mathrm{nmol}$ miR-28-5p agomir $(\mathrm{n}=4)$ or NC $(\mathrm{n}=4)$. (B) Tumor size was measured at different conditions. (C) The tumor weight in nude mice with miR-28-5p agomir treatment was statistically significant compared with $\mathrm{NC}\left({ }^{* *} \mathrm{P}<0.01\right)$. (D) The tumor volume in nude mice with miR-28-5p agomir treatment was statistically significant compared with NC. 
cell apoptosis in vitro. Moreover, miR-28-5p promoted the growth of ovarian tumor in vivo.

Epithelial-mesenchymal transition (EMT) is the core of normal embryonic development, and a biological process that the epithelial cells are translated into the mesenchymal phenotype cells (40). It has played an important role in the development of embryonic, chronic inflammation, tissue reconstruction, cancer metastasis, and many fibrotic diseases (41). In the process of EMT, a main characteristic is that the expression level of cell adhesion molecules (E-cadherin) was decreased; and the the expression level of mesenchymal markers (such as vimentin) was increased (23). Some studies indicated that EMT has very important effect in tumor invasion and metastasis (42). Therefore, the process of EMT is related to invasion, metastasis and drug resistance of tumors. In this study, we indicated that miR-28-5p downregulated the protein expression level of E-cadherin and upregulated the protein expression level of vimentin in ES2 and SKOV3 cells. Therefore, our studies demonstrated that miR-28-5p promotes the progress of EMT in ovarian carcinoma cells. Previous studies have shown that F-actin is interrelated with the mobility, contraction, and migration of cells $(24,43)$. Our results indicated that miR-28-5p increased the protein expression level of F-actin. This demonstrated that miR-28-5p may promote the progress of migration in ovarian cancer cells.

N4BP1 is closely related to the normal development by acting on the related E3 ligase ITCH (26). It was supposed that N4BP1 may have ribonuclease activity because the YacP-like nuclease (NYN) domain possesses nucleases (28). Therefore, N4BP1 plays a major role in gene regulation. However, the function and mechanism research of N4BP1 have not been reported in ovarian cancer. Therefore, in this study, we indicated that the expression level of N4BP1 was lower in ovarian cancer tissues compared with normal ovarian tissues. miR-28-5p inhibited the mRNA expression level of N4BP1 in ES2 and SKOV3 cells. Therefore, we came to a conclusion that miR-28-5p downregulated N4BP1 expression in human ovarian cancer. This further revealed the important roles of N4BP1, which miR-28-5p promoted in the development and progression of ovarian cancer through inhibition of N4BP1.

In conclusion, this study indicated that miR-28-5p and N4BP1 had high expression in ovarian cancer tissues. miR-28-5p promoted the progression of ovarian cancer cell cycle, proliferation, migration and invasion, and inhibited apoptosis in vitro and promoted the growth of ovarian tumor in vivo. miR-28-5p promoted the progress of EMT in ovarian carcinoma cells. In addition, we indicated that miR-28-5p downregulated N4BP1 expression in human ovarian cancer. This further revealed that miR-28-5p promoted the development and progression of ovarian cancer through inhibition of N4BP1. This study may provide the potential theoretical foundation for the diagnosis and treatment of ovarian cancer.

\section{Acknowledgements}

This study was supported by Natural Science Foundation of Guangdong Province (nos. S2012010006150 and S2012040006148) and Science and Technology Planning Project of Guangdong Province, China (no. 2014A020212710).

\section{References}

1. Siegel R, Naishadham D and Jemal A: Cancer statistics, 2012. CA Cancer J Clin 62: 10-29, 2012.

2. Siegel R, Ward E, Brawley O and Jemal A: Cancer statistics, 2011: The impact of eliminating socioeconomic and racial disparities on premature cancer deaths. CA Cancer J Clin 61: 212-236, 2011.

3. Iorio MV, Visone R, Di Leva G, Donati V, Petrocca F, Casalini P, Taccioli C, Volinia S, Liu CG, Alder H, et al: MicroRNA signatures in human ovarian cancer. Cancer Res 67: 8699-8707, 2007.

4. Zaman MS, Maher DM, Khan S, Jaggi M and Chauhan SC: Current status and implications of microRNAs in ovarian cancer diagnosis and therapy. J Ovarian Res 5: 44, 2012.

5. Heintz AP, Odicino F, Maisonneuve P, Quinn MA, Benedet JL, Creasman WT, Ngan HY, Pecorelli S and Beller U: Carcinoma of the ovary. FIGO 26th Annual Report on the Results of Treatment in Gynecological Cancer. Int J Gynaecol Obstet 95 (Suppl 1): S161-S192, 2006.

6. Bartel DP: MicroRNAs: Genomics, biogenesis, mechanism, and function. Cell 116: 281-297, 2004.

7. Iorio MV and Croce CM: MicroRNAs in cancer: Small molecules with a huge impact. J Clin Oncol 27: 5848-5856, 2009.

8. Farazi TA, Hoell JI, Morozov P and Tuschl T: MicroRNAs in human cancer. Adv Exp Med Biol 774: 1-20, 2013.

9. Djuranovic S, Nahvi A and Green R: A parsimonious model for gene regulation by miRNAs. Science 331: 550-553, 2011.

10. Kasinski AL and Slack FJ: Epigenetics and genetics. MicroRNAs en route to the clinic: Progress in validating and targeting microRNAs for cancer therapy. Nat Rev Cancer 11: 849-864, 2011.

11. Baer C, Claus R and Plass C: Genome-wide epigenetic regulation of miRNAs in cancer. Cancer Res 73: 473-477, 2013.

12. Di Leva $G$ and Croce CM: The role of microRNAs in the tumorigenesis of ovarian cancer. Front Oncol 3: 153, 2013.

13. Nam EJ, Yoon H, Kim SW, Kim H, Kim YT, Kim JH, Kim JW and Kim S: MicroRNA expression profiles in serous ovarian carcinoma. Clin Cancer Res 14: 2690-2695, 2008.

14. Dahiya N and Morin PJ: MicroRNAs in ovarian carcinomas. Endocr Relat Cancer 17: F77-F89, 2010.

15. Mateescu B, Batista L, Cardon M, Gruosso T, de Feraudy Y, Mariani O, Nicolas A, Meyniel JP, Cottu P, Sastre-Garau X, et al: miR-141 and miR-200a act on ovarian tumorigenesis by controlling oxidative stress response. Nat Med 17: 1627-1635, 2011.

16. Dahiya N, Sherman-Baust CA, Wang TL, Davidson B, Shih IeM, Zhang Y, Wood W III, Becker KG and Morin PJ: MicroRNA expression and identification of putative miRNA targets in ovarian cancer. PLoS One 3: e2436, 2008.

17. Hell MP, Thoma CR, Fankhauser N, Christinat Y, Weber TC and Krek W: miR-28-5p promotes chromosomal instability in VHL-associated cancers by inhibiting Mad2 translation. Cancer Res 74: 2432-2443, 2014.

18. Almeida MI, Nicoloso MS, Zeng L, Ivan C, Spizzo R, Gafà R, Xiao L, Zhang X, Vannini I, Fanini F, et al: Strand-specific miR-28-5p and miR-28-3p have distinct effects in colorectal cancer cells. Gastroenterology 142: 886-896.e9, 2012.

19. Li Q, Zhu F and Chen P: miR-7 and miR-218 epigenetically control tumor suppressor genes RASSF1A and Claudin-6 by targeting HoxB3 in breast cancer. Biochem Biophys Res Commun 424: 28-33, 2012.

20. Creighton CJ, Gibbons DL and Kurie JM: The role of epithelialmesenchymal transition programming in invasion and metastasis: A clinical perspective. Cancer Manag Res 5: 187-195, 2013.

21. Lamouille S, Xu J and Derynck R: Molecular mechanisms of epithelial-mesenchymal transition. Nat Rev Mol Cell Biol 15: 178-196, 2014.

22. Sheppard D: Epithelial-mesenchymal interactions in fibrosis and repair. Transforming growth factor- $\beta$ activation by epithelial cells and fibroblasts. Ann Am Thorac Soc 12 (Suppl 1): S21-S23, 2015.

23. Fraga CH, True LD and Kirk D: Enhanced expression of the mesenchymal marker, vimentin, in hyperplastic versus normal human prostatic epithelium. J Urol 159: 270-274, 1998.

24. McGough A, Pope B, Chiu W and Weeds A: Cofilin changes the twist of F-actin: Implications for actin filament dynamics and cellular function. J Cell Biol 138: 771-781, 1997.

25. Murillas R, Simms KS, Hatakeyama S, Weissman AM and Kuehn MR: Identification of developmentally expressed proteins that functionally interact with Nedd4 ubiquitin ligase. J Biol Chem 277: 2897-2907, 2002. 
26. Oberst A, Malatesta M, Aqeilan RI, Rossi M, Salomoni P, Murillas R, Sharma P, Kuehn MR, Oren M, Croce CM, et al: The Nedd4-binding partner 1 (N4BP1) protein is an inhibitor of the E3 ligase Itch. Proc Natl Acad Sci USA 104: 11280-11285, 2007.

27. Sharma P, Murillas R, Zhang H and Kuehn MR: N4BP1 is a newly identified nucleolar protein that undergoes SUMO-regulated polyubiquitylation and proteasomal turnover at promyelocytic leukemia nuclear bodies. J Cell Sci 123: 1227-1234, 2010.

28. Anantharaman V and Aravind L: The NYN domains: Novel predicted RNAses with a PIN domain-like fold. RNA Biol 3 . 18-27, 2006.

29. Jiang L, Lai YK, Zhang J, Wang H, Lin MC, He ML and Kung HF: Targeting S100P inhibits colon cancer growth and metastasis by Lentivirus-mediated RNA interference and proteomic analysis. Mol Med 17: 709-716, 2011.

30. Madhyastha HK, Radha KS, Nakajima Y, Omura S and Maruyama M: uPA dependent and independent mechanisms of wound healing by C-phycocyanin. J Cell Mol Med 12B: 2691-2703, 2008.

31. Cannistra SA: Cancer of the ovary. N Engl J Med 351: 2519-2529, 2004.

32. Greenlee RT, Hill-Harmon MB, Murray T and Thun M: Cancer statistics, 2001. CA Cancer J Clin 51: 15-36, 2001.

33. Wang J, Paris PL, Chen J, Ngo V, Yao H, Frazier ML, Killary AM, Liu CG, Liang H, Mathy C, et al: Next generation sequencing of pancreatic cyst fluid microRNAs from low grade-benign and high grade-invasive lesions. Cancer Lett 356B: 404-409, 2015.

34. Stahlhut $\mathrm{C}$ and Slack FJ: MicroRNAs and the cancer phenotype: Profiling, signatures and clinical implications. Genome Med 5 : $111,2013$.
35. Cheng W, Liu T, Wan X, Gao Y and Wang H: MicroRNA-199a targets CD44 to suppress the tumorigenicity and multidrug resistance of ovarian cancer-initiating cells. FEBS J 279: 2047-2059, 2012.

36. Nagaraja AK, Creighton CJ, Yu Z, Zhu H, Gunaratne PH Reid JG, Olokpa E, Itamochi H, Ueno NT, Hawkins SM, et al: A link between mir-100 and FRAP1/mTOR in clear cell ovarian cancer. Mol Endocrinol 24: 447-463, 2010.

37. Fu X, Tian J, Zhang L, Chen Y and Hao Q: Involvement of microRNA-93, a new regulator of PTEN/Akt signaling pathway, in regulation of chemotherapeutic drug cisplatin chemosensitivity in ovarian cancer cells. FEBS Lett 586: 1279-1286, 2012.

38. Zhou SL, Hu ZQ, Zhou ZJ, Dai Z, Wang Z, Cao Y, Fan J, Huang XW and Zhou J: miR-28-5p-IL-34-macrophage feedback loop modulates hepatocellular carcinoma metastasis. Hepatology 63: 1560-1575, 2016.

39. Shi $X$ and Teng F: Down-regulated miR-28-5p in human hepatocellular carcinoma correlated with tumor proliferation and migration by targeting insulin-like growth factor-1 (IGF-1). Mol Cell Biochem 408: 283-293, 2015.

40. Hanahan D and Weinberg RA: Hallmarks of cancer: The next generation. Cell 144: 646-674, 2011.

41. Kalluri R and Weinberg RA: The basics of epithelial-mesenchymal transition. J Clin Invest 119: 1420-1428, 2009.

42. De Wever O, Demetter P, Mareel M and Bracke M: Stroma myofibroblasts are drivers of invasive cancer growth. Int $\mathbf{J}$ Cancer 123: 2229-2238, 2008.

43. Lauffenburger DA and Horwitz AF: Cell migration: A physically integrated molecular process. Cell 84: 359-369, 1996. 